\title{
May Body Adiposity Index be used as adiposity diagnostic marker?
}

\section{Abstract}

Body Adiposity Index (BAI) is the method of determining body fat content based on anthropometric measurements, proposed as an alternative to the BMI. Due to the fact that the hip circumference is taken into account in calculating BAI, it is suggested that this index better reflects the differences in women and men obesity than BMI. The aim of this study was to examine the association between BAI and other adiposity indices such as WHtR (Waist to Height Ratio), Body Mass Index (BMI), Waist Circumference (WC), sum of skinfolds, or adiposity tissue measured by bioelectrical impedance analysis (FM-BIA) among adults, independence of sex and age. The study group consisted of 336 adults (59\% women, 41\% men) aged 18-70 years, which was divided into two groups: 18-45 years $(57 \%)$ and $>45(43 \%)$ to further analysis. All anthropometric measurements: height (H), body weight (BW), WC, hip circumference (HC) skinfolds thickness were taken according to the standardized procedures, with light clothing and without shoes twice and averages were calculated. BIA was performed under standardized conditions according to the manufacturer's protocol (Maltron BioScan 920 ver.1.1). BAI was calculated as $\left[\mathrm{HC}(\mathrm{cm}) / \mathrm{H}(\mathrm{m})^{1.5}-18\right]$. Linear regression was used to investigate the relationship between BAI and adiposity indices. BAI was positively associated with sex, age, BW, WC, FM-BIA, BMI, sum of 4 or 5 skinfolds and WHtR for all subjects, but the strongest with FM-BIA $(\beta=0.787, \mathrm{p}<0.001)$, BMI $(\beta=0.696$, p $<0.001)$ or WHtR $(\beta=0.625, p<0.00001)$ for total group. BAI correlation with BW, WC, FM-BIA, BMI or WHtR were stronger in women than in men, both for the total group and regards to age. The identified set of correlates explained (for above variables) $30-79 \%$, of the total variation in BAI for women, and $15-49 \%$ for men. The stronger BAI correlation with sex was found in the older group ( $>$ 45 years old $)$ than in younger $(\beta=0.582, p<0.00001$ vs. $\beta=0.372, p<0.00001)$. In men aged $>45$ years, stronger BAI correlations with the sum of 4 or 5 skinfolds were observed. The correlation between BAI and BW in men was weaker than in women regardless of age $(\beta=0.364, \mathrm{p}<0.00001$ vs. $\beta=0.762, \mathrm{p}<0.00001)$. Considering strong correlation between BAI and fat content (FM-BIA), BMI, WHtR, WC and BW in women, it seems that BAI may be used to determine the content of adipose tissue or as a diagnostic marker, but in men its using need further research.

\section{Conflict of Interest}

There is no conflict of interest 